\title{
Sub-keV ring current ions as the tracer of substorm injection
}

\author{
M. Yamauchi and R. Lundin \\ Swedish Institute of Space Physics, SE-98128 Kiruna, Sweden
}

Received: 29 June 2005 - Revised: 24 November 2005 - Accepted: 21 December 2005 - Published: 7 March 2006

\begin{abstract}
The dynamics of the energy-latitude dispersed sub-keV trapped ions inside the ring current region, the so-called wedge-like dispersions structure, were statistically studied using Viking satellite data. Probabilities with/without these signatures at various local times in the dayside are obtained in terms of different time-lags from the substorm activity monitored by the $A E$ index. The structure appears in the early morning sector within a few hours after the substorm, and it slowly propagates eastward while decaying with a time scale of several hours. The result qualitatively confirmed the previous model that the wedge-like dispersions are originated from past substorm-related plasma injections into the nightside ring current region, and that the dispersion is formed when these injected plasma slowly moves eastward to the dayside by the drift motion $(\boldsymbol{E} \times \boldsymbol{B}$ (eastward), grad- $|B|$ (westward), and curvature (westward) drifts). However, the appearance of the structure is twice or three times faster than the model prediction, and some structure reaches even to the evening sector. The results indicate that the start location of the drift is not as far as midnight and that the drift speed is slightly faster than the model prediction. The former means that the substorm-related increase of hot plasma in the ring current region shifts or extends to the early morning sector for large substorms, and the latter means that the substantial electric field driving the sub-keV ion drift is slightly different from the model field. We also detected the evacuating effect starting right after the substorm (or storm) onset. The electric field imposed in the dayside magnetosphere seems to remove the remainder of trapped ions.
\end{abstract}

Keywords. Magnetospheric physics (Energetic particles, trapped; Plasma convection; Storms and substorms)

\section{Introduction}

Satellites traversing inside the dayside ring current region frequently detect wedge-like energy-latitude dispersed dense trapped sub-keV ions far equatorward of the auroral region (Yamauchi et al., 1996a, 2005; Ebihara et al., 2001). They

Correspondence to: M. Yamauchi

(m.yamauchi@irf.se) are clearly separated from the energetic component of the ring current, and are well recognized in almost all satellite data as a zoo of various dispersion patterns. To understand the origin of these sub-keV plasma, Ebihara et al. (2001) made a particle drift simulation using up to 2 nd order invariant conservation for both the $\boldsymbol{E} \times \boldsymbol{B}$ drift (eastward, energy independent) and $\operatorname{grad}-|B| /$ curvature drifts (westward for positive ions, energy dependent). The simulation assumed bursty (order of tens of minutes) injection of Maxwellian plasma from the nightside, presumably during substorms, into the dipole-like magnetic field region, and it successfully reconstructed the majority of the patterns of energy-latitude dispersions, as well as their local time dependence (Fig. 5 of Yamauchi et al., 2005). Although Ebihara et al. (2001) did not identify the actual source mechanism, all three cases simulated in the paper are traced back to the midnight sector during the high $A E$ period.

The simulation result suggests that these particles originate from past substorm-related injection of dense, hot plasma into the nightside ring current region about $5 \sim 20 \mathrm{~h}$ earlier. In other words, the observed wedge-like energylatitude dispersed sub-keV ions may directly reflect nightside electromagnetic disturbances. In this sense, they are probably the same as the regionally isolated sub-keV plasma sheetlike ions precipitating in the plasmasphere (Shelley et al., 1972; Sauvaud et al., 1981; Chappel et al., 1982; Newell and Meng, 1986). The latter is also considered to be of substorm origin.

Using the Aureol-1 satellite (altitude $400 \sim 2500 \mathrm{~km}$ ), Sauvaud et al. (1981) demonstrated that sub-keV ion precipitation in the subauroral region increases some hours after substorm injection in the early morning (00-06 magnetic local times (MLT)) sector. Using low-altitude DMSP F6 and F7 satellites, Newell and Meng (1986) demonstrated that sub-keV ion precipitation in the late morning subauroral region ( 08:30 MLT) is strongly correlated with the $K_{p}$ index with some hours of time delay, and the enhanced precipitation may last a day. Both observations are in good agreement with the simulation by Ebihara et al. (2001).

Unfortunately, no solid data analyses exist to confirm the relationship between the wedge-like structure and the substorm activity. The purpose of this paper is to examine this 


\section{Viking positive ion $\left(\mathrm{i}^{+}\right)$data Orbits 1112 1115 1986-9-12 (00 14 UT)}
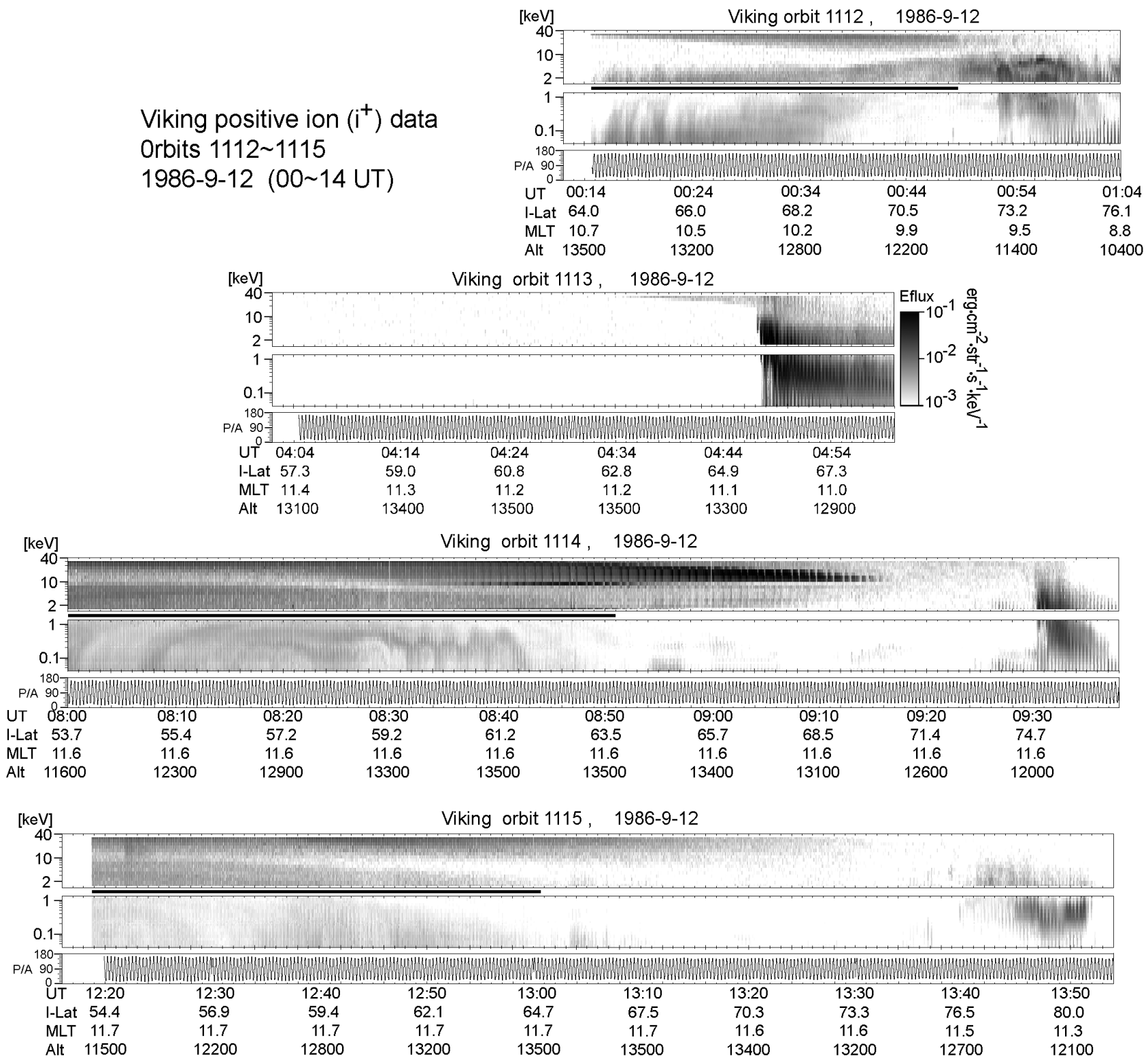

Fig. 1. Viking energy-time spectrograms for positive ions energy flux $(0.05-40 \mathrm{keV})$ during four consecutive traversals of the dayside ring current region on 12 September 1986 (orbits 1112 to 1115). The region with the dense trapped sub-keV ions of our interest is indicated by the thick line between panels.

relation and to obtain statistically the first-order values of the drift time and decay time. We use sub-keV ion data from the Swedish Viking satellite (March-October 1986) because the $A E$ index is available from all 12 stations. $A E$ values after 1990 are obtained with limited stations and hence there is a slight risk of missing localized substorms.

\section{A case study}

As Yamauchi et al. (2005) demonstrated, the identification of the wedge-like structures at different altitudes is not yet well established. This is partly because the high-altitude satellites seem to reflect the temporal development of the phe- nomenon during a single traversal, and partly because the mirror altitude of these particles is comparable to the altitude of the low-altitude satellite. Therefore, we must examine mid-altitude satellite data for the present purpose for which the simulation by Ebihara et al. (2001) is made. The other requirement for the data set is that sub-keV ion data with good pitch-angle coverage is available during the meridional traversal over the outer radiation belt. The Viking satellite satisfies these requirements. Only Akebono and FAST satellites can provide a similar quality of data set as Viking, while complete $A E$ from all 12 stations is available only for the Viking period. Viking provides $8000 \mathrm{~km}-13000 \mathrm{~km}$ altitude data for $50 \mathrm{eV}-40 \mathrm{keV}$ ions and electrons for an 8-month period. 
If the orbital period is much shorter than the lifetime of the phenomenon, the best method is to compare consecutive traversals before, during, and after substorms and make statistics. Unfortunately, Viking's orbital period of about $4.6 \mathrm{~h}$ is comparable to the lifetime of the phenomenon. Hence, such a comparison of consecutive traversals is quite difficult because it requires one traversal during the middle of the isolated activity and the other consecutive travels during the quiet period, before and after the activity.

Figure 1 shows one of the best examples of consecutive traversals of Viking for a case study of the temporal variation. The sub-keV ion data from four consecutive Viking pre-noon traversals (all at around 11:30 11:40 MLT except orbit 1112 at $\sim 10: 30$ MLT) on 12 September 1986 is displayed. All four traversals covered latitudinal ranges wide enough to examine the existence of structures inside the ring current region. This sequence is during the initial phases of the largest magnetic storm in the entire Viking data set. The geomagnetic indices during this period are shown in Fig. 2.

While data from orbit 1113 (coverage $>58$ invariant latitude (ILat)) does not show any signature of sub-keV ions equatorward of the boundary layer (here we use a broad sense of "boundary layer" either with magnetosheath-like ions or $\mathrm{keV}$ ions with structures, see Woch and Lundin, 1993, and Kremser and Lundin, 1990, for details), the traversals before and after it show clear energy-latitude dispersed trapped sub$\mathrm{keV}$ ions several degrees equatorward of the boundary layer. The dense trapped sub-keV ions are observed all the way up to $\sim 71$ ILat in orbit 1112 (data coverage $>64$ ILat), up to $\sim 64$ ILat in orbit 1114 (data coverage $>54$ ILat), and up to $\sim 66$ ILat in orbit 1115 (data coverage $>54$ ILat). The extra internal structure seen in orbit 1114 is further studied in Yamauchi et al. (1996b).

The traversal with the dense sub-keV ions is found after the largest substorm, whereas the traversal during the peak of the substorm does not show any signature. This superficial anti-correlation is, however, qualitatively consistent with the simulation by Ebihara et al. (2001). In other words, this single case can be interpreted in many different ways. Therefore, we should take a different approach, i.e. a statistical method, such as the superposed epoch analyses, using the entire Viking data.

\section{Analyses method}

We employ backward superposed epoch analysis, i.e. we find out the timing (in hours) of the nearest substorm activity before the Viking traversals, and arrange all Viking traversals in terms of given time-lags from the nearest substorm activity before the traversal. Since the time-lag is supposed to be different at different MLT sectors, this superposed epoch analysis is made for each MLT sector. Here, we counted the number of Viking traversals with/without the wedge-like structure for each bin that is organized by both MLT and timelags, from the latest substorm judged from the $A E$ index.
Geomagnetic Indices 11 12 September, 1986

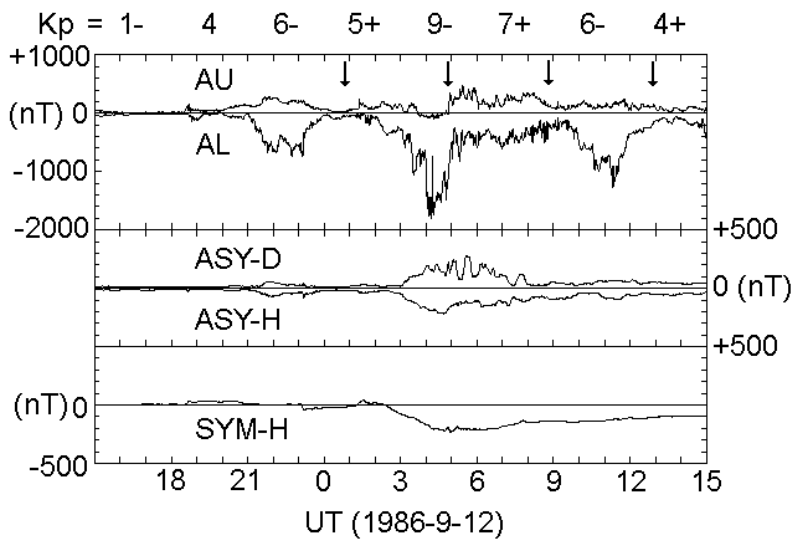

Fig. 2. Geomagnetic indices (AU, AL, SYM, and ASY) during and before the traversals displayed in Fig. 1.

Then we obtained the occurrence probability of the wedgelike dispersed sub-keV ions at different 2-D bins.

If the wedge-like structure is the "fossil" of substorm activity, the probability of observing the wedge-like structure must be a smooth function of the time-lag from the substorm and of the MLT. In this case, the final plot, organized by the time-lag for a fixed MLT sector, should give us characteristic times of drift and decay. Furthermore, the peak probability of observing the structure can be obtained for each MLT sector. The drift time from the nightside obtained at each MLT gives the drift speed and hence the effective electric field by comparison with the simulation result.

Strictly speaking, the hot plasma injection and $A E$ are not related one-to-one to each other, because different types of injections appear at different phases of substorms (e.g. injections related to substorm onset and the overshoot of the plasma flow that refills the plasma sheet during the recovery phase; please refer to Ebihara et al. (2001) for other scenarios). However, we do not have to know the phase of the substorm that produces the hot plasma in the low-L shell in the present purpose because the substorm duration $(\sim$ tens of minutes) is shorter than the drift time and the decay time (expected to be $\sim$ a few to ten hours, and it actually is according to the results). We just need the accuracy in the order of hours.

Considering the fact that Viking takes nearly one hour to traverse the subauroral region where the wedge-like structure is found, using hourly values of $A E$ is sufficient. Furthermore, one cannot discuss the accuracy less than one hour when simply using the $A E$ values without specifying a phase of substorms as the epoch. Yet two types of epoch are used as the substorm activities in this paper, the end of the activities, and the start of activities, as illustrated in Fig. 3.

Figure 3a shows an ideal case with an isolated $A E$ activity with the duration shorter than the drift time. If such cases represent the majority, the backward superposed epoch analysis may provide many characteristic times, including 

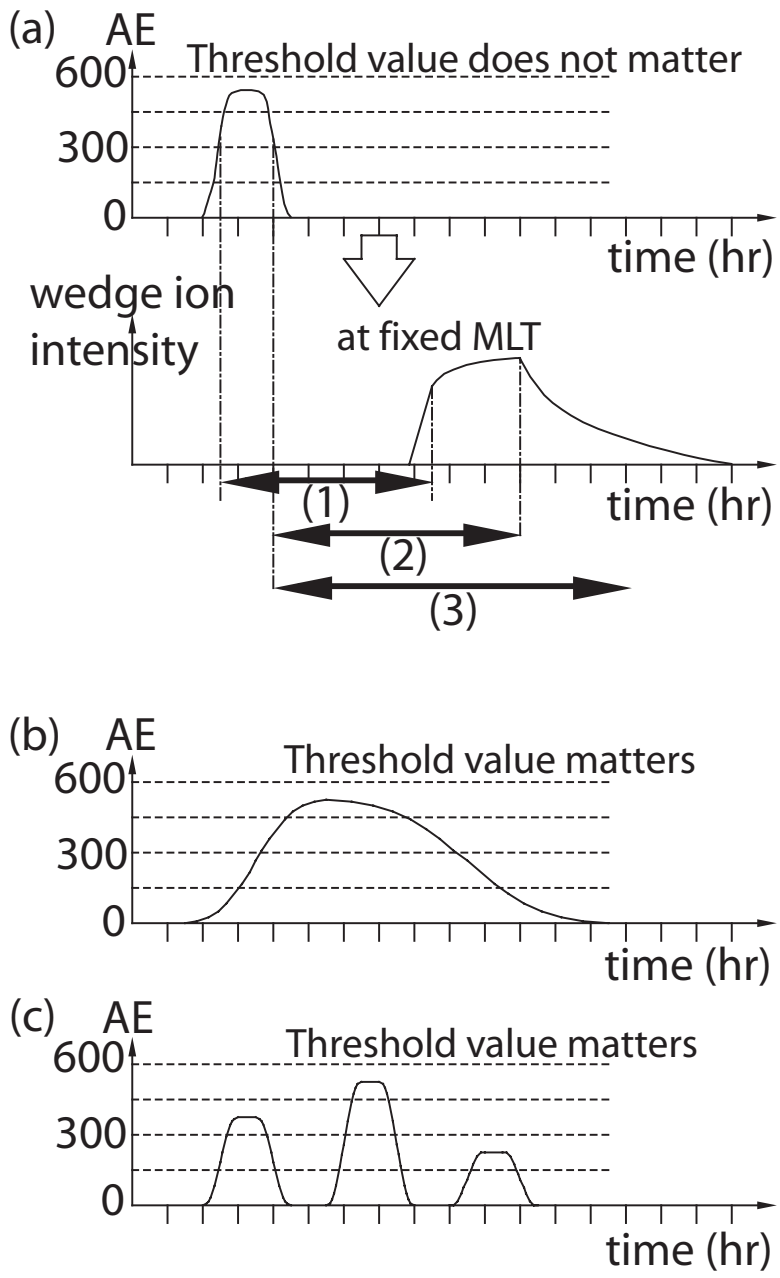

Fig. 3. Illustration of simplified histories of the hot plasma injection from the nightside, as characterized by $A E$, and the expected intensity of the wedge-like structure in the dayside at a fixed local time. (a) The ideal case is that only one isolated substorm occurred within 1 day before the traversal, and that the substorm duration is shorter than the drift time. (b) The actual increase/decrease of $A E$ can be more gradual, and the injection might gradually increase or decrease. (c) Even worse, we often observe more than two activities with short quiet intervals in between. The arrows in (a) indicates three characteristic times to be considered: (1) drift time of the injected sub-keV ions; (2) propagation time of the last injected ions; (3) decay time of the sub-keV ions added to (2).

three basic values illustrated in the figure. (1) Drift time of the injected sub-keV ions is given by the time-lag when the probability of observing the wedge-like structure suddenly increases (in statistics, this is the reaching point to a plateau) after the start of the activity. (2) Drift time of the last injected ions is given by the time-lag when the probability of observing the wedge-like structure suddenly decreases (in statistics, this is the end point from a plateau) after the end of the activity. (3) Decay time of the sub-keV ions added to (2) is the time to reach the asymptotic probability.
Figure $3 \mathrm{~b}$ shows a case when the increase/decrease of $A E$ is gradual, i.e. when the injection gradually increases or decreases. In this case, we have to define proper threshold values of $A E$, one value corresponding to the start of the major injection and the other corresponding to end of the last injection. This case produces a spread of values in characteristic times, but we can still obtain the most probable values.

Figure $3 \mathrm{c}$ shows an even more complicated situation when we observe more than two $A E$ activities with a relatively short quiet period in between. In this case, setting the threshold value is important in obtaining the proper time-lag. If the last activity does not produce injections, we have to set the threshold high enough to exclude the last activity from the analysis. However, if the last activity is responsible for the observed wedge-like structure, we have to set the threshold low enough to include the last activity in the analysis. The worst case is when the first activity is responsible for the observed signature. Then, there is no way to obtain the correct time-lag because the time-lag in our method is registered either from the second activity (for high threshold case) or from the last activity (for low threshold case). Of course making a statistical analysis reduces this problem, but only if the data set is sufficiently large. For the limited data of Viking, it is difficult to estimate how much the result is distorted.

The problem with these nonideal profiles (Figs. $3 b$ and c) is minimized if we take the time-lag from the end of the activity instead of the start of the activity, because, if so, we do not have to worry too much about whether the first activity generates sub-keV ion injections or not in the last case (Fig. 3c). Thus, the backward superposed epoch analysis is more reliable if the epoch is defined as the end of the activity (see cases (2) and (3) in Fig. 3) than the start of the activity (see case (1) in Fig. 3). In both cases, the probability curve after the first peak is less reliable than the probability curve until the first peak, because the probability curve mixes many effects: the substorm history and duration, injection location, and the decay process.

For the analysis against the start of the activity, it is advisable to have a slightly higher threshold value than the end threshold because we examine slightly different matters, i.e. the propagation of the "end of injection" (or "no injection") from the end of the activity, and the propagation of the "start of injection" (or "with injection") from the start of the activity. No matter how high we set the start threshold, the average time-lag from the start of the activity tends to be shorter than the actual value, due to the problem mentioned above (cf. Fig. 3c). Therefore, obtaining the shortest characteristic time (duration (1) in Fig. 3) is less problematic than obtaining longer characteristic times (duration (2) and (3) in Fig. 3) when using the start of the activity as the epoch.

In this paper we took the threshold values of hourly $A E>300 \mathrm{nT}$ as the end of the substorm and $>400 \mathrm{nT}$ as the start of the substorm, or more specifically, the activity level that produces the substorm-related increase of hot plasma in the ring current region (most likely due to injections from the tail plasma sheet). We tried various threshold values from 
$100 \mathrm{nT}$ to $500 \mathrm{nT}$, and the result is qualitatively the same, except that the derived characteristic time and the peak probabilities are $A E$ dependent. Studies with various $A E$ threshold values will be presented in a different report.

The duration of active hours defined by hourly $A E$ is normally only $1 \sim 2 \mathrm{~h}$. Nearly half, i.e. $55 \%(45 \%)$ of the hourly $A E>400 \mathrm{nT}(>300 \mathrm{nT})$ activities in this study, ended with only $1 \mathrm{~h}$, while $28 \%(30 \%)$ of the hourly $A E>400 \mathrm{nT}$ $(>300 \mathrm{nT}$ ) activities lasted only $2 \mathrm{~h}$. The average duration of $>400 \mathrm{nT}$ hourly $A E$ activity is less than $2 \mathrm{~h}$ and it is about $2 \mathrm{~h}$ for $>300 \mathrm{nT}$ hourly $A E$ activity. Therefore, we do not have to worry too much about the prolonged activity that is illustrated in Fig. 3b.

For any probability analyses, one must identify null cases. To judge if a traversal is quiet (e.g. orbit 1113 in Fig. 1) or not, the most strict way is to limit the database to traversals that cover the entire region where the wedge-like structure can be seen. However, not too many traversals satisfy this condition. Therefore, we use all Viking traversals that cover at least latitudes $>67$ ILat, with at least 4 deg in width equatorward of the boundary-type ion region. This criterion is statistically adequate in identifying quiet traversals, because among 373 traversals with some signature of wedgelike structure, 351 traversals (94\%) show the structure either at latitudes $>67$ ILat or within 4 deg from the equatorward end of the boundary region. Examples in Fig. 1 (orbits 1114 and 1115) are two of the exceptional 22 traversals. In this way we increased the database to more than 700 traversals.

To maintain good statistics, we also (1) limited the traversals to the dayside (05-19 MLT), where Viking has relatively good coverage, (2) adapted 3-h MLT bins instead of 1-h MLT bins (each 3-h MLT bin contains more than 90 traversals), (3) took 3-h windows (running summation) for the time-lag, except for 0-th hour and 1-st hour, (4) added all types of dispersions that are predicted by the simulation by Ebihara et al. (2001), and (5) divided the traversals into only three categories: with dispersed dense sub-keV ions (hereafter called "clear structure"), with some sub-keV ions but less clear (hereafter called "marginal"), and with no or very faint signatures of such sub-keV ions (hereafter called "quiet" traversals).

The variety of dispersion patterns and their characteristic energies (we added all patterns) make the identification difficult by numerical algorithms. We therefore identified the wedge-like structures by visual inspection using the following criteria: a clear case comprises more than about 2-deg latitudinal range of broadly distributed sub-keV plasma with count rates roughly more than 30 ; and a marginal case comprises more than about 1-deg latitudinal range of broadly distributed sub-keV plasma with count rates roughly more than 10 (five is the noise level for Viking). With this classification, the MLT dependence without the subdivision of the dispersion patterns is given in Fig. 4. For subdivided statistics, please refer Fig. 5 of Yamauchi et al. (2005).

It is worth noting the relation between the wedgelike dispersion and the $D_{s t}$ index. Out of 202 clearcase traversals, 179 traversals $(89 \%)$ are within $12 \mathrm{~h}$ after

\section{Occurrence of wedge-like structure}

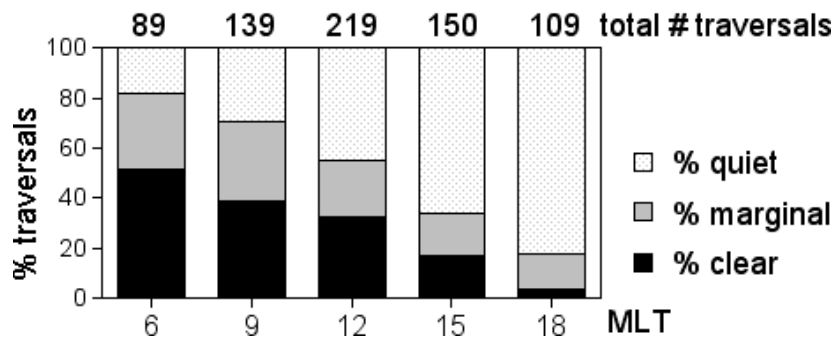

Fig. 4. Local time distribution of the wedge-like dispersion signature observed by Viking (1986.3-1986.10). We took the probability of identifying phenomena during a traversal which covers at least $>67$ ILat. The black bar (lower part), gray bar (middle part), and white bar (upper part) denote the traversals with a clear signature of structured dense sub-keV ions, with some structures but not strong signature, and with very weak or no signature, respectively. The number on the top of each bar is the total number of traversals in each MLT bin.

hourly $A E>300 \mathrm{nT}$ activities and 129 traversals (64\%) are within $12 \mathrm{~h}$ after hourly $A E>400 \mathrm{nT}$ activities, whereas only 55 traversals $(27 \%)$ are within $12 \mathrm{~h}$ after $D_{s t}<-30 \mathrm{nT}$ activities and 11 traversals $(5 \%)$ are within $12 \mathrm{~h}$ after $D_{s t}<-60 \mathrm{nT}$ activities. Obviously, the magnetic storm activity $\left(D_{s t}\right.$ activity) is not the major cause of the wedge-like structure in the ring current region, although the structure is located in the ring current region.

\section{Result}

Figure 5 shows the probabilities of traversals with clearly identifiable structure (black bar), and with some structure but less clear, i.e. the marginal cases (gray bar), and the quiet traversals (dotted bar) as a function of time-lag from the end of the active $A E$ period. The threshold value for the active level is set as hourly $A E>300 \mathrm{nT}$. Results from five MLT sectors are presented from top to bottom. The horizontal axis is the time-lag from the end of the activity.

Here, we examine the probability of quiet traversals, or more precisely, the time-lag that gives the minimum probability of quiet traversals. This is equivalent to the time-lag to reach the maximum probability of observing some signature, and corresponds to case (2) of Fig. 3, i.e. the typical drift time of the last injected plasma. Since no hot plasma injection is expected in the source region after the epoch, we expect a decrease in probability of observing a wedge-like structure after the last plasma passed through the given MLT. Equivalently, an increase in this probability (or decrease in quiet probability) means that the "end of injection" information has not yet arrived. The latter (decrease of quiet probability) is observed at the initial hours for all MLT.

Similarly, the time-lag to reach the final (maximum) probability corresponds to case (3) of Fig. 3, i.e. a summation of propagation time and decay time. The same logic should be applied to the time profile for clear cases, but the trend is 

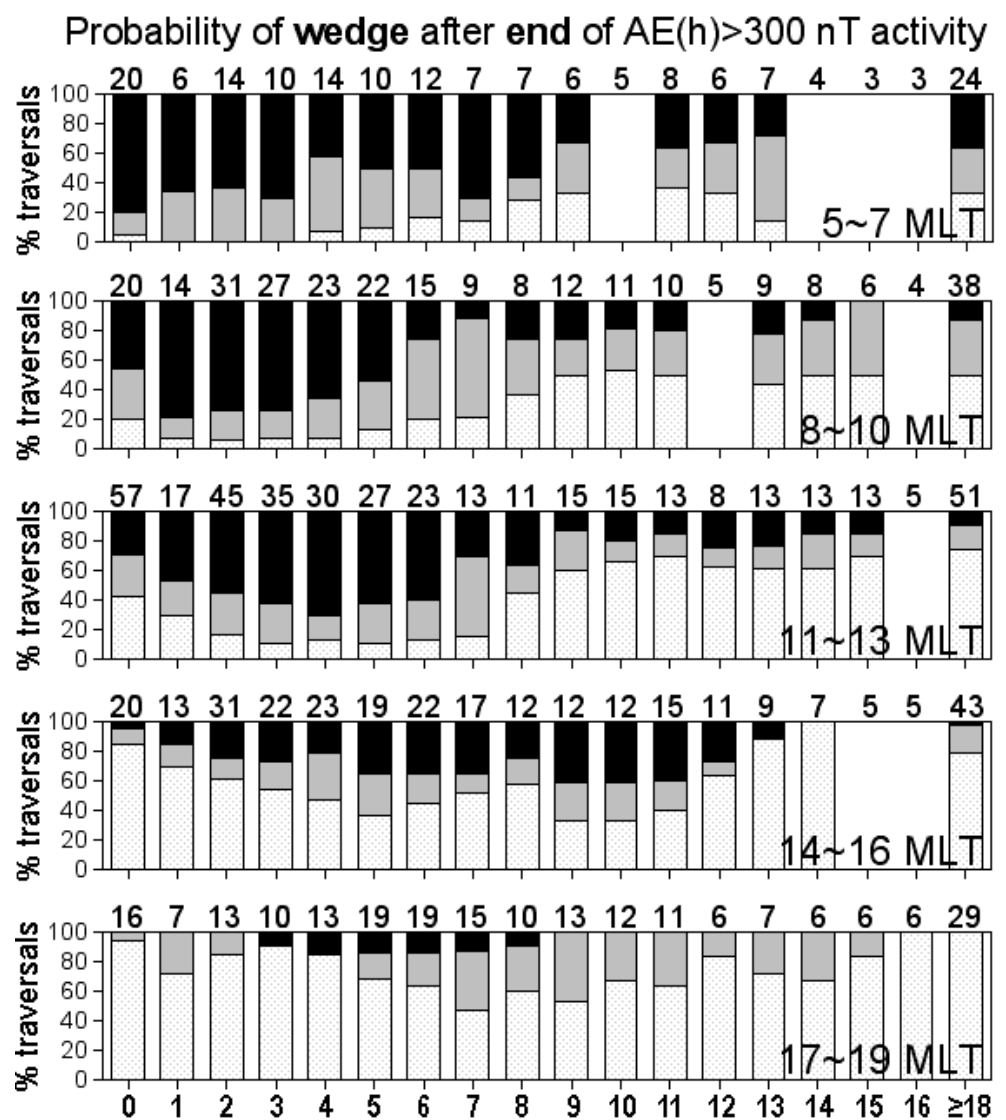

hours ( $\Sigma$ 3-hour window) from end of latest hourly $A E>300 \mathrm{nT}$ activity

$\geq$ traversals/bin $\quad \square$ quiet
marginal case

- clear case

Fig. 5. Probabilities of observing a wedge-like structure for different time-lags from the end of the high $A E$ period (hourly $A E>300 \mathrm{nT}$ ). From top to bottom, 05 07 MLT, 08 10 MLT, 11 13 MLT, 14 16 MLT, and 17 19 MLT. In each panel, the black bar, gray bar, and white bar denote the traversals with clear signature of structured dense sub-keV ions, with some structure but not strong signature, and with very weak or no signature, respectively. To increase statistics, a 3-h window (running summation) is taken, and the horizontal axis shows the central time-lag from the end of activity. For example, the time-lag of $5 \mathrm{~h}$ means that the actual time-lag is 4,5 , or $6 \mathrm{~h}$, i.e. the hourly $A E$ has been less than $300 \mathrm{nT}$ for at least $4 \mathrm{~h}$ before the traversal but it was more than $300 \mathrm{nT}$ at 5 , 6, or $7 \mathrm{~h}$ before the traversal. For the time-lag of $1 \mathrm{~h}$ and $0 \mathrm{~h}$, we did not take such a running summation (window is $1 \mathrm{~h}$ ). The last column denotes all traversals that the time-lag is equal to or more than $18 \mathrm{~h}$. The bins with less than 6 traversals are not shown. The total number of traversals in each 3-h bin is written at the top of each bar.

not as clear as what we obtain from the quiet case in Fig. 5. Therefore, we examine the probability of quiet traversals.

Let us first examine Fig. 5 for a fixed local time, for example, in the late morning (08-10 MLT) sector. The probability of quiet traversals decreases from $20 \%$ to $5 \%$ during the first $2 \mathrm{~h}$ after the end of the $300 \mathrm{nT}$ hourly- $A E$ activity, and starts increasing from the 4 th hour toward an asymptotic (maximum) value of about 50\% around $9 \mathrm{~h}$ after the end of the activity. Probabilities of quiet traversals at the other MLT sectors show similar profiles, i.e. they first decrease for a few to several hours, to reach minimum values, and then increase again toward asymptotic (maximum) values. Thus, we see a certain relation between the appearance of the wedge-like structure and the hourly $A E$ activity.

Next, we compare the time profile at different local times. The lowest probabilities of quiet traversals are: about $0 \%$ at
$1 \sim 3 \mathrm{~h}$ after the end of the $300 \mathrm{nT}$ hourly- $A E$ activity at $05-$ 07 MLT, about 5\% at 2 3h for 08-10 MLT, about $10 \%$ at $3 \sim 5 \mathrm{~h}$ for $11-13 \mathrm{MLT}$, about $35 \%$ at $4 \sim 6 \mathrm{~h}$ for $14-16 \mathrm{MLT}$, and about $50 \%$ at $6 \sim 8 \mathrm{~h}$ for $17-19$ MLT. The asymptotic probabilities of quiet traversals are: about $30 \%$ at $8 \sim 9 \mathrm{~h}$ after the end of the $300 \mathrm{nT}$ hourly- $A E$ activity at 05-07 MLT, about $50 \%$ at $9 \sim 10 \mathrm{~h}$ for $08-10 \mathrm{MLT}$, about $70 \%$ at $10 \sim 11 \mathrm{~h}$ for 11-13 MLT, about $80 \%$ at $12 \sim 13 \mathrm{~h}$ for 14-16 MLT, and about $100 \%$ at $14 \sim 16 \mathrm{~h}$ for $17-19$ MLT. The result indicates an eastward movement of minimum quiet probability and a common decay time of about 6 or $7 \mathrm{~h}$ for all MLT sectors.

Although the eastward movement of the minimum and asymptotic probabilities of the quiet traversal is clear, the probability itself is always higher at larger MLT at any hour after the end of activity. In other words, the peak of the probability of observing the wedge-like structure is always 


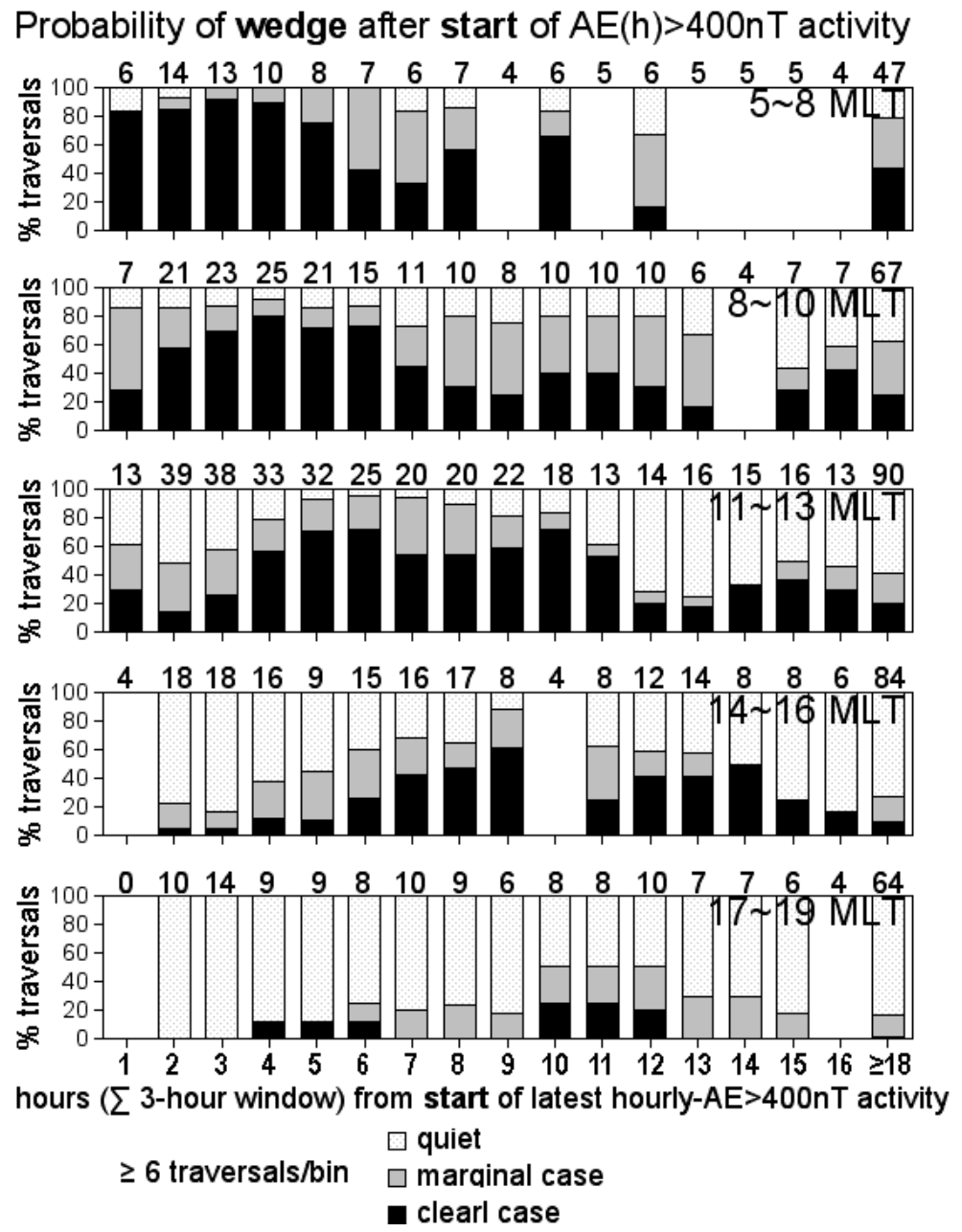

Fig. 6. The same as the previous figure, except that the time axis represents the time-lag from the start of the latest activity with hourly $A E>400 \mathrm{nT}$.

highest in the morning sector at any time. This is not the case with the probability of clear cases. For example, at the 4th and 5th hours in Fig. 5, the highest probability is registered at noon (11-13 MLT) instead of the morning (05-07 MLT). This "azimuthal shift of the peak" becomes clearer if we examine the probability of the wedge-like structure after the start of the latest activity.

Figure 6 shows probabilities of traversals with/without the wedge-like structure as a function of time-lag after the start of the latest active $A E$ period in the same format as Fig. 5. This time the threshold of hourly $A E$ is set to $400 \mathrm{nT}$, and we directly examine the probability of traversals with clear structure because this analyses corresponds to case (1) of Fig. 3.

Let us again first examine a fixed (08-10 MLT) sector. The probability of observing clear structures quickly increases until the 4 th hour, reaching about $75 \%$. It remains rather constant until the 6th hour, and finally begins to decay. The sharp increase toward the largest probability is also seen in the other MLT sectors. The resultant peak is found at a larger time-lag at a larger MLT, indicating that these sub-keV ions are slowly drifting eastward.
As discussed in the previous section, some substorms may produce injections before the hourly $A E$ reach $400 \mathrm{nT}$ (cf. Figs. $3 b$ and $c$ ). For these cases the wedge-like structure is observed at a shorter time-lag than it should be, and in statistics this effect appears as the gradual increase of the probability. This is one of the reasons why the probability increases already at the first hour in the morning sectors. Therefore, the drift time corresponds to the time-lag when the probability reaches the plateau. It is about $2 \sim 4 \mathrm{~h}$ at $08-10 \mathrm{MLT}$, with a peak probability of $75 \%$. Similarly, one can obtain the characteristic drift time to $11-13$ MLT as $4 \sim 6 \mathrm{~h}$, with a peak probability of $70 \%$, and to $14-16$ MLT as $6 \sim 7 \mathrm{~h}$, with a peak probability of $50 \%$.

In the morning sector (05-07 MLT), the probability reached a peak value of $85 \%$ already at the 1 st hour. This suggests that the threshold value of $400 \mathrm{nT}$ is too high: this sector is so close to the source region that plasma injections from weak substorms may reach this MLT before decaying. Therefore, one can only say that the drift time is less than $3 \mathrm{~h}$. The situation is opposite for the evening sector (17-19 MLT), where we see a very minor peak at the 5th hour and a normal peak at the 13th hour, with a peak probability of $25 \%$. 
Table 1. Characteristic times (hours) and probability (\%).

\begin{tabular}{cccc}
\hline MLT & $\begin{array}{c}\text { lowest quiet } \\
(* 1)\end{array}$ & $\begin{array}{c}\text { asymptotic quiet } \\
(* 2)\end{array}$ & $\begin{array}{c}\text { highest clear } \\
(* 3)\end{array}$ \\
\hline $05 \sim 07$ & $1 \sim 3 \mathrm{~h}(0 \%)$ & $8 \sim 9 \mathrm{~h}(30 \%)$ & $0 \sim 3 \mathrm{~h}(85 \%)$ \\
$08 \sim 10$ & $2 \sim 3 \mathrm{~h}(5 \%)$ & $9 \sim 10 \mathrm{~h}(50 \%)$ & $2 \sim 4 \mathrm{~h}(75 \%)$ \\
$11 \sim 13$ & $3 \sim 5 \mathrm{~h}(10 \%)$ & $10 \sim 11 \mathrm{~h}(70 \%)$ & $4 \sim 6 \mathrm{~h}(70 \%)$ \\
$14 \sim 16$ & $4 \sim 6 \mathrm{~h}(35 \%)$ & $12 \sim 13 \mathrm{~h}(80 \%)$ & $6 \sim 7 \mathrm{~h}(50 \%)$ \\
$17 \sim 19$ & $6 \sim 8 \mathrm{~h}(50 \%)$ & $14 \sim 16 \mathrm{~h}(100 \%)$ & $10 \mathrm{~h}(25 \%)$ \\
\hline
\end{tabular}

(*1) Minimum probability of quiet case after the end of a $300 \mathrm{nT}$ activity.

(*2) Asymptotic probability of quiet case after the end of a $300 \mathrm{nT}$ activity.

(*3) Maximum probability of clear case after the start of a $400 \mathrm{nT}$ activity.

Although the first peak is caused by a single traversal and the second peak is caused by two traversals, it is worth noting that the first peak disappears and the second peak is enhanced if we set the $A E$ threshold value to $500 \mathrm{nT}$, i.e. all three traversals fall into the same time-lag for a high $A E$ threshold value.

Thus, the optimum $A E$ threshold value is most likely different at different MLT sectors with higher values at larger MLT. We tried different $A E$ threshold values and $500 \mathrm{nT}$ gives the most concentrated profile only for 17-19 MLT, whereas $200 \mathrm{nT}$ gives the most concentrated profile for $05-$ 07 MLT. A full analysis with various $A E$ threshold values will be presented in a different report.

In the noon (11-13 MLT) to afternoon (14-16 MLT) sectors, the probability of observing the wedge-like dispersed sub-keV ions first decreases during the first couple of hours. Note that the first several hours in the noon sectors have statistically sufficient traversals in each bin and this decrease is most likely real. The minimum probability is even lower than the asymptotic probability after $18 \mathrm{~h}$. Such a decrease in probability at the initial few hours is seen even if we change the $A E$ threshold values. Therefore, these decreases might not simply be the natural decrease reflecting the decay of previous activity, but most likely reflect a forced removal of particles during the initial phase of new activity.

In fact, Fig. 1 shows such an effect. The traversal of orbit 1113 at the noon sector took place only $6 \mathrm{~h}$ after the previous strong substorm with hourly $A E>700 \mathrm{nT}$ (and $5 \mathrm{~h}$ after hourly $A E>300 \mathrm{nT}$ activity). According to Fig. 5, this condition predicts $60 \%$ probability of observing a clear structure and only $10 \%$ probability of it being quiet. Yet orbit 1113 shows a completely quiet traversal. This "null" observation strongly supports the hypothesis that sub-keV ions are effectively removed when very strong substorms take place.

Table 1 summarizes the result. The drift time obtained from the quiet case (Fig. 5) and that from the clear cases (Fig. 6) are generally consistent with each other, although the drift of the start of the activity is slightly slower than that of the end of the activity in the afternoon sector. This indicates that the "end of injection" (or "last injection") in- formation propagates in the same way as the "start of the injection" information. This equivalence is pointed out in Ebihara et al. (2001): the wedge-like pattern is seen even for the "empty" region between the plasma cloud with a sharp boundary as the internal structure (see their Fig. 2).

Table 1 clearly shows that the peak of the probability of observing clear wedge-like structures is moving eastward. Although we can recognize the decay of the peak as time progresses, the peak is actually moving. By comparing with Fig. 4, the peak probability of observing clear wedge-like structures is $30 \sim 40 \%$ higher than the all-time probability for 05-16 MLT, and it is more than 5 times higher in the evening sector. The decay time at each MLT is given as the difference in time-lags between the asymptotic quiet probability and the minimum quiet probability. It is $6 \sim 8 \mathrm{~h}$ at all MLT. Although the present method makes the decay time less reliable than the drift time as mentioned in Sect. 3, the consistency over different MLT sectors makes the obtained decay time reliable.

Combining the MLT dependence reported in Yamauchi et al. (2005), we can draw a picture of how the wedge-like dispersion slowly drifts eastward after a substorm, as illustrated in Fig. 7. As it travels eastward, the wedge-like dispersed trapped sub-keV plasma also changes its morphology as well as its intensity.

\section{Discussion}

As mentioned in conjunction with Fig. 3 where only simplified cases are considered, we have problems in defining the "end" and "start" of the substorm activity that are responsible for the observed wedge-like structures during each traversal. While the problem with the "end" of the activity can be solved by testing various threshold values of hourly $A E$, the problem with the "start" of the activity in the case of multiple peaks (cf. Fig. 3c) requires correlation analysis or forward superposed epoch analysis instead of the backward superposed epoch analysis. However, with a data set of about 700 traversals (100 200 traversals at each MLT sector), the statistical error is larger than the difference between methods, particularly for the present purpose to derive the characteristic times shown in Fig. 3a.

With this limitation in mind, let us compare the present result with the predictions by the particle drift simulation by Ebihara et al. (2001). According to the drift model, the quiet probability (or the "end of the last injection", as explained in Sect. 3) should start increasing monotonically after a short time-lag in the early morning sector and after a longer time-lag at a later local time, because it takes some hours for the last injected sub-keV ions to reach there by the drift. In addition, the minimum quiet probability (or maximum probability to detect some structures) should increase toward the evening sector because some wedge-like structure already stagnates in the morning sector when the magnetospheric electric field is weak. This feature is enhanced by 


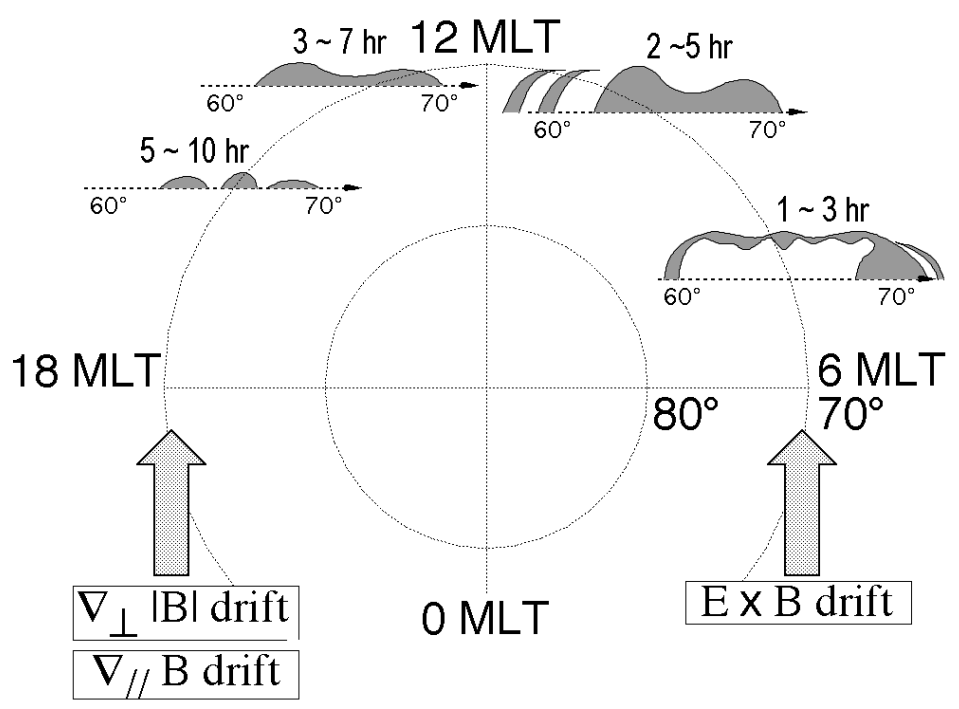

Fig. 7. A summary illustration of MLT dependence of the wedge-like structure for both the morphology and typical time-lags from the substorm activity.

decay processes such as the charge exchange. Both features are seen in Fig. 5.

The simulation also predicts slow eastward motions of the peak probabilities for clear cases while decaying during the motion. This is exactly what we see in Fig. 6. Thus, the summary picture illustrated in Fig. 7 is consistent with the simulation by Ebihara et al. (2001), except that the observed morphology is more complicated and the traveling time is shorter than the simulation result. With this result, the wedge-like dispersed sub-keV ions are most likely the same as the isolated ion precipitation at low altitudes reported by Sauvaud et al. (1981) and Newell and Meng (1986). In other words, the source population of these precipitating ions is most likely trapped ions, if observed at mid-altitude.

There are some additional features in the observation. First, the wedge-like structure drifts eastward beyond what the model predicts. The majority of the activities with hourly $A E>400 \mathrm{nT}$ reached the noon sector, and nearly half of them reached the early afternoon sector. The structure can travel even to the evening ( $>17$ MLT) sector. Figure 6 strongly indicates that the wedge-like structures observed in the evening sector drift from the morning rather than directly from the evening. If they come directly from the nightside via westward drift, they should appear at much earlier hours (Fig. 7 of Ebihara et al., 2001).

Second, the observed drift times are much shorter than the values given in the simulation by one-half to one-third. This difference is partly attributed to the fact that the appearance of the structure at 06 MLT is nearly immediate. In simulation the elapse time to 06 MLT is several hours. Subtracting this effect, the actual drift velocity in the dayside is much closer to the model prediction. Since the simulation by Ebihara et al. (2001) used the empirical convection electric field model (Volland-Stern type electric field with parameter determined from observed average convection (Volland, 1973;
Table 2. $\Omega_{V} / \Omega_{\text {cor }}(* 1)$ from empirical model.

\begin{tabular}{cccc}
\hline & $K_{p}=2$ & $K_{p}=3$ & $K_{p}=4$ \\
\hline $\mathrm{L}=4$ & 0.08 & 0.13 & 0.25 \\
$\mathrm{~L}=6$ & 0.25 & 0.4 & 0.8 \\
\hline
\end{tabular}

(*1) Angular velocity by the $E \times B$ drift (cf. Eq. 1)

Stern 1975; Maynard and Chen, 1975)) with dipole field, the drift speed itself can be roughly estimated from a simple calculation.

The Volland-Stern type electric potential $\Phi$ is given by $\Phi=A R^{2} \sin \phi$, where $R$ is the radial distance from the center of the Earth, $\phi$ is the MLT, $A$ is the intensity of the convection electric field, which depends on the magnetospheric activities, e.g., $A=0.12 \mathrm{kV} / R_{E}^{2}$ for $K_{p}=2, A=0.2 \mathrm{kV} / R_{E}^{2}$ for $K_{p}=3$, and $A=0.33 \mathrm{kV} / R_{E}^{2}$ for $K_{p}=4$, and $R_{E}$ is the Earth's radius. Neglecting the grad- $|B|$ and curvature drifts (very small for sub-keV ions), we obtain the azimuthal component of the $\boldsymbol{E} \times \boldsymbol{B}$ drift velocity as

$(\boldsymbol{E} \times \boldsymbol{B})_{\phi} / B^{2}=2 A R \sin \phi / B$.

Assuming the dipole magnetic field (same as the simulation), we obtain the azimuthal drift angular velocity $\Omega$ as

$$
\begin{aligned}
\Omega & =\Omega_{c o r}+\Omega_{V} \sin \phi \\
\Omega_{V} & =2 A\left(R / R_{E}\right)^{3} / B_{0},
\end{aligned}
$$

where $\Omega_{\text {cor }}=1 \mathrm{LT} / \mathrm{h}$ is the corotation angular velocity, $\Omega_{V} \sin \phi$ is the contribution from the Volland-Stern type electric field, and $B_{0}$ is the magnetic field strength on the Earth's surface. Table 2 lists the contribution from the latter at $L=4 \sim 6$, i.e. at latitudes where the wedge-like structures 
are observed. Note that the majority of the period relevant to the present statistics was under the condition of $K_{p} \leq 4$.

On the other hand, the observed values of $\Omega-\Omega_{\text {cor }}$ are: $\sim 1 \mathrm{LT} / \mathrm{h}$ at $06 \sim 09$ MLT $(\sin \phi=0.7 \sim 1), 0.5 \sim 1 \mathrm{LT} / \mathrm{h}$ at $09 \sim 15$ MLT $(\sin \phi=-0.7 \sim 0.7)$, and $\ll 0.5 \mathrm{LT} / \mathrm{h}$ at $15 \sim 18$ MLT ( $\sin \phi=-1 \sim-0.7$ ), according to Table 1 . These velocities are now close to the model velocity in Table 2, but they are still slightly higher than the model values. We cannot explain this discrepancy in this paper, and we simply list our speculations for the cause of the faster drift in the observation than the model prediction: (a) The electric field has a radial component inward (this makes the sin factor nonzero at noon). (b) The electric field is slightly stronger than the model. (c) The drifting path is at a high $\mathrm{L}$ shell $(\mathrm{L}>6)$. (d) The bulk effect of plasma plays a role, i.e. the plasma tries to keep its momentum in the same way as the plasma transfer event (Lundin et al., 2003).

Third, the decay time is the same at all MLT sectors and is several hours. This value is consistent with the charge exchange lifetime. The charge exchange lifetime for keV protons is several hours, and sub-keV ions are expected to have a slightly longer lifetime (S. Barabash, private communication, 2005), while the lifetime of oxygen ions is much longer. Since the wedge-like structure consists of both protons and oxygen ions, the total decay time is expected to be several to ten hours, with the lowest energy signature remaining the longest at the post-noon sector. This is exactly what is observed. Note that the loss by pitch-angle scattering into the loss cone is much less effective than the charge exchange loss (Newell and Meng, 1986).

The decay time obtained is slightly longer than the convection time, i.e. the time that the structure comes into a given MLT sector and drifts away from there. Therefore, we expect that the decrease in the probability of observing the wedgelike structure should also show a "convecting" behavior. This is also observed if one looks at the probability of clear cases in Fig. 5. For example, at the 4th and 5th hours, the highest probability is registered at noon (11-13 MLT) instead of in the morning (05-07 MLT). The result for the clear case (left panel) indicates that the dense population of trapped sub-keV ions is convecting. This becomes clearer if we examine the probability of the wedge-like structure after the start of the latest activity (Fig. 6). On the other hand, we obtained the higher probability of the quiet traversal at larger MLT all the time in Fig. 5. Therefore, the wedge-like structure is not simply moving as a whole, but some ions (either a part of the structure or the entire structure for some events) remain and decay in the morning sector or late morning sector.

Fourth, we observed the evacuation of the wedge-like structured sub-keV ions during the initial $1 \sim 2 \mathrm{~h}$ of the substorm or storm at pre-noon sectors. Such an evacuation effect has already been reported in late $1970 \mathrm{~s}$ for $>0.3 \mathrm{keV}$ trapped electrons and $\mathrm{keV}$ ions in a series of works by Hultqvist and his coworkers (e.g. Hultqvist et al., 1981). They suggested that this evacuation is caused by the newly-developed substorm-related electric field at mid-altitude, as well as at low-altitude. Recently, C:son Brandt et al. (2002) showed some case studies using the energetic neutral particle imaging technique in which $>10 \mathrm{keV}$ ring current ions in the dayside were evacuated on a time scale of $10 \mathrm{~min}$ at the beginning of the substorm.

Fifth, the response at 06 MLT is nearly immediate $(0 \sim 3 \mathrm{~h})$. This indicates that the source of the drifting plasma (or destination of the substorm injections) extends to the early morning sector, e.g. 04 05 MLT. Such an extended injection region is possible for large substorms, and if so, our result should be somewhat dependent on the $A E$ threshold. In fact, the optimum $A E$ threshold values are different for different local times, with higher values at later local times as mentioned in conjunction with Fig. 6.

The immediate response in the morning sector, the quick appearance in the noon sectors, and the MLT dependence of the optimum $A E$ threshold, indicate that the closest source to the dayside is located in the early morning sector for large substorms. This also explains why we did not observe the second peak (see two paragraphs below) at 17 19 MLT. The next question concerns exact source distribution: whether a single large substorm causes such a widespread source region or each substorm causes a narrow source region with the source location shifted toward morning for large substorms. Here we note that the quick response is also seen after the end of the activity, i.e. for the last injection. This fact means that nothing remains at around 03 MLT for some substorms (particularly large ones) at time-lag $0 \mathrm{~h}$. Therefore, the latter scenario is most likely. The former scenario requires a mechanism that prevents the sub-keV ions at the midnight ring current region from drifting to the morning sector for only large substorms.

One possible scenario is as follows. Since a substorm that reaches hourly $A E>500 \mathrm{nT}$ can probably produce an electric field strong enough to inject sub-keV ions to the early morning sector, it starts drifting while the magnetospheric electric field is still high. A weak substorm with $\sim 300 \mathrm{nT}$ probably produces injections only to the midnight sector, and the magnetospheric electric field is already very weak when it reaches the morning sectors. Of course, one may also point out another factor, i.e. the magnetospheric electric field (and hence the drift speed) is probably larger after a larger $A E$ activity in our data set. To examine these scenarios we need further analyses, together with the $A E$ dependence.

Finally, we note the double peaks of the clear-case probability. As discussed in Sect. 3 (cf. Fig. 3c), the profile after the first peak mixes the substorm history, injection location, and the decay process. Because our database is rather limited, it is not wise to discuss the details of the time profile of the probability beyond the first peak. With this limitation in mind, we would yet like to point out that the clear-case probabilities have double peaks with the peak-to-peak interval of about 5 6h for all MLT, except 17-19 MLT. Such a consistent interval may reflect some systematic causes. Note that the double peak appears only in the clear-cases, but not in the total of the clear and marginal cases.

The substorm-related increase of the hot plasma in the ring current region (e.g. by injection) most likely widely 
distributes in longitude, as discussed above. Since the drift from the pre-midnight sector $(21 \sim 23 \mathrm{MLT})$ to the early morning sector $(04 \sim 05 \mathrm{MLT})$ takes about $5 \sim 6 \mathrm{~h}$ at $\mathrm{L}=4$ according to Table 2 , the double peak appears if the source region is distributed along these MLT with the highest probability at the early morning sector and the pre-midnight sector.

On the other hand, the double peak might reflect the active period's duration (Fig. 3b) or interval (e.g. Fig. 3c) if such an interval or duration has a characteristic time. The duration of the active period is less than $2 \mathrm{~h}$ in a majority of the cases, and it cannot systematically make the double peak with $5 \sim 6 \mathrm{~h}$ of separation. On the other hand, the most frequent peak-to-peak intervals between hourly $A E>400 \mathrm{nT}$ (while it decrease $<200 \mathrm{nT}$ in between) is $7 \sim 8 \mathrm{~h}$ according to 10-year hourly $A E$ data (1978-1987). Note that this is different from the interval between substorms $(2.75 \mathrm{~h}$, Borovsky et al., 1993) because the hourly value represents the average activity rather than individual substorms. The most frequent interval between active hourly $A E$ periods matches the interval between the double peaks.

As discussed with Fig. 3c, there are two situations with possible offsets: (1) the time-lag is registered shorter than the actual one when the latest $A E$ peak above the threshold ( $>400 \mathrm{nT}$ ) is not responsible for the observed wedge; (2) the time-lag is registered longer than the actual one when the latest $A E$ peak responsible for the observed wedge is below the threshold. For the first case, the second peak of the clear-case probability reflects the responsible activity and the first peak reflects the "ghost" activity without the wedge-like structure. Then, the second peak should not be lower than the first peak, but this is not the case in Fig. 6. The first peak of the clear-case probability is always higher than the second peak.

For the second case, the most probable time-lag to resister is after the characteristic repetition time mentioned above, and hence the second peak of the clear-case probability may appear after the substorm intervals. In this case the double peak should be weakened if we set a low threshold value. We found such an $A E$ threshold dependence, but the analysis of the result requires lengthy discussion and should be presented in a separate paper. On the other hand, the peak-topeak intervals between hourly $A E$ activities distributes in a Poisson-like fashion, without outstanding peak values. Only $10 \%$ of intervals ( $3 \%$ in hours) falls into the peak-to-peak intervals of $7 \sim 8 \mathrm{~h}$ between the active hourly $A E$ period, and $30 \%$ ( $8 \%$ in hours) are less than $10 \mathrm{~h}$. With this smooth distribution, it is difficult to produce a systematic second peak in the probability plot. Even if it could produce the second peak, such a peak should appear as the result of large statistics. In this sense the second peak is caused by the limited statistics even if the interval between the active periods might have caused the double peak.

We leave this problem for the future work, together with the $A E$ dependence. For such studies, it is ideal if we introduce more classifications in terms of types of dispersion, $A E$ threshold (e.g. narrow banding of the $A E$ value by introducing upper cutoff in addition to lower cutoff), and 10-min average values of $A E$ instead of 60-min average values. Unfortunately, such an analysis requires a much larger database than the Viking database.

\section{Conclusions}

Using Viking sub-keV ion data and $A E / D_{s t}$ indices we examined the dynamic change in the location of the wedgelike dispersion structures after substorm activities. The result is summarized in Table 1 and Fig. 7, and is consistent with simulations by Ebihara et al. (2001); i.e. sub-keV ions originated from the nightside during substorms, drift eastward, and cause the wedge-like dispersion structures that slowly propagate eastward while decaying. Therefore, the source population of precipitating ions in the subauroral region, separated from auroral region (e.g. Sauvaud et al., 1981; Newell and Meng, 1986), are most likely the same as the observed wedge-like dispersed trapped ions at mid-altitude. In addition, we found:

1. The wedge-like structure is related to the past $A E$ activity but not directly to the past or present $D_{s t}$ activity, although it is located in the ring current region.

2. The wedge-like structure drifts eastward beyond what the model predicts. The majority of the activities with hourly $A E>400 \mathrm{nT}$ drive the structure to the noon sector, and nearly half of them reach the early afternoon sector.

3. The structures seen in the evening sector have most likely traveled from the morning sector by eastward drift rather than directly from the nightside by westward drift.

4. The response at 06 MLT is nearly immediate $(0 \sim 3 \mathrm{~h})$ after the high $A E$ activities. This indicates that the source of the drifting plasma (or destination of the substorm injections) shifts or extends to the early morning, e.g. 04 05 MLT. Obtaining the exact distribution requires a further $A E$-dependence study.

5. The drift times to the late morning sectors (few hours) and to the noon sector (several hours) for the activities with hourly $A E>400 \mathrm{nT}$ are twice or three times shorter than model predictions. Even by taking into account the morning-shift of source, the derived drift speed is somewhat faster than model prediction.

6. The optimum $A E$ threshold value to obtain the drift result is most likely different at different MLT with higher values at larger MLT.

7. The decay time of several hours at all MLT is consist with the charge exchange lifetime.

8. Sub-keV ions are sometimes evacuated right after the onset of a substorm or storm. The electric field imposed on the dayside seems to remove the remainder of the trapped ions. 
Acknowledgements. The Viking project is sponsored by the Swedish National Space Board. The $A E, D_{s t}$, SYM, and ASY indices are provided by WDC-C2 for geomagnetism at Kyoto University, and the $K_{p}$ index is provided by Geophysical Institute at Göttingen University. We thank Y. Ebihara for useful comments. M. Yamauchi thanks programs for disabled people in Sweden which have made it possible for him to work. The paper commemorates 20 years anniversary of Viking that was launched February 1986.

Topical Editor T. Pulkkinen thanks P. T. Newell and Y. Ebihara for their help in evaluating this paper.

\section{References}

Borovsky, J. E., Nemzek, R. J., and Belian, R. D.: The occurrence rate of magnetospheric-substorm onsets: Random and periodic substorms, J. Geophys. Res., 98, 3807-3814, 1993.

C:son Brandt, P., Mitchell, D. G., Ebihara, Y., Sandel, B. R., Roelof, E. C., Burch, J. L., and Demajistre, R.: Global IMAGE/HENA observations of the ring current: Examples of rapid response to IMF and ring current-plasmasphere interaction, J. Geophys. Res., 107(A11), 1359-1370, doi:10.1029/2001JA000084, 2002.

Chappell, C. R., Green, J. L., Johnson, J. F. E., and Waite Jr., J. H.: Pitch angle variations in magnetospheric thermal plasma: Initial observations from Dynamic Explorer-1, Geophys. Res. Lett., 9, 933-936, 1982.

Ebihara, Y., Yamauchi, M., Nilsson, H., Lundin, R., and Ejiri, M.: Wedge-like dispersion of sub-keV ions in the dayside magnetosphere: Particle simulation and Viking observation, J. Geophys. Res., 106, 29 571-29 584, 2001.

Hultqvist, B., Aparicio, B., Borg, H., Arnoldy, R., and Moore, T. E.: Decrease of $\mathrm{keV}$ electron and ion fluxes in the dayside magnetosphere during the early phase of magnetospheric disturbances, Planet. Space Sci., 29, 107-126, 1981.

Kremser, G. and Lundin, R.: Average spatial distributions of energetic particles in the midaltitude cusp/cleft region observed by Viking, Geophys. Res., 95, 5753-5766, 1990.
Lundin, R., Sauvaud, J.-A., Rème, H., Balogh, A., Dandouras, I., et al.: Evidence for impulsive solar wind plasma penetration through the dayside magnetopause, Ann. Geophys., 21, 457-472, 2003,

SRef-ID: 1432-0576/ag/2003-21-457.

Maynard, N. C. and Chen, A. J.: Isolated cold plasma regions: Observations and their relation to possible production mechanisms, J. Geophys. Res., 80, 1009-1013, 1975.

Newell, P. T. and Meng, C. I.: Substorm introduction of $1 \mathrm{keV}$ magne-tospheric ions into the inner plasmasphere, J. Geophys. Res., 91, 11 133-11 145, 1986.

Sauvaud, J. A., Crasnier, J., Mouala, K., Kovrazhkin, R. A., and Jorjio, N. V.: Morning sector ion precipitation following substorm injections, J. Geophys. Res., 86, 3430-3438, 1981.

Shelley, E. G., Johnson, R. G., and Sharp, R. D.: Satellite observations of energetic heavy ions during a geomagnetic storm, J. Geophys. Res., 77, 6104-6110, 1972.

Stern, D. P.: The motion of a proton in the equatorial magnetosphere, J. Geophys. Res., 80, 595-599, 1975.

Volland, H.: A semiempirical model of large-scale magnetospheric electric fields, J. Geophys. Res., 78, 171-180, 1973.

Woch, J. and Lundin, R.: The low-latitude boundary layer at midaltitudes: Identification based on Viking hot plasma data, Geophys. Res. Lett., 20, 979-982, 1993.

Yamauchi, M., Lundin, R., Eliasson, E., and Norberg, O.: Mesoscale structures of radiation belt/ring current detected by lowenergy ions, Adv. Space Res., 17, (2), 171-174, 1996a.

Yamauchi M., Lundin, R., Mursula, K., Marklund, G., and Potemra, T. A.: Dayside Pc5 pulsation detected by Viking ion data at $\mathrm{L}=4$, Geophys. Res. Lett., 23, 2517-2520, 1996b.

Yamauchi, M., Lundin, R., Eliasson, L., Winningham, D., Rème, H., et al.: Structures of sub-keV ions inside the ring current region, in: The Inner Magnetosphere: Physics and Modeling, edited by: Pulkkinen, T. I., Tsyganenko, N. A., and Friedel, R., AGU Monoraph, 41-46, 2005. 